\title{
AN EFFICIENT STATISTICAL MULTIPLEXING METHOD FOR H.264 VBR VIDEO SOURCES FOR IMPROVED TRAFFIC SMOOTHING
}

\author{
T.Raghuveera and K.S.Easwarakumar \\ Department of Computer Science and Engineering, Anna University, Chennai, India. \\ \{traghuveera, easwara\}@cs.annauniv.edu
}

\begin{abstract}
Frame level H.264/MPEG encoded VBR video traffic is highly bursty in nature because of inherent coding techniques employed. Multiplexing traffic from many VBR sources results in smoothing of generated traffic from the multiplexer, and improves Statistical Multiplexing Gain (SMG). An efficient multiplexing methodology can greatly enhance resource utilization. Performance of multiplexer can be estimated by addressing the burstiness and statistical multiplexing gain. We present here a new multiplexing method named "ERA multiplexing", which is quite simple, faster and efficient as opposed to any other known methods like, Frame-aligned multiplexing, Frame-lag based multiplexing and random multiplexing. Our experiments have proved that ERA method is much superior in terms of smoothing the traffic and achieving better statistical multiplexing gain. We have tested the technique with high quality frame size traces of Star Wars-IV encoded using H.264/SVC and H.264/AVC to justify our claims.
\end{abstract}

\section{KEYWORDS}

VBR video, Burstiness, H.264, Statistical Multiplexing Gain, traffic smoothing.

\section{INTRODUCTION}

With the ever growing demand for network resources, allocation of resources for a bursty frame size VBR video traffic from MPEG/H.264 encoders is quite challenging. Providing end-to-end Quality of Service is a hectic task for a service provider. Isochronous traffic has strict QoS constraints throughout the channel usage. It is often the case that, a resource (say bandwidth) allocated to a source is either under utilized or over utilized, resulting in unexpected losses, as well as quality deflections. Popular video coding standards like MPEG, H.264/AVC (Advanced Video Coding), H.264/SVC (Scalable Video Coding), have successfully addressed the issue of size versus quality. For a given video quality, H.264SVC has the least size while MPEG has considerably larger size, H.264/AVC stands inbetween. However this tradeoff has had its impact on the "burstiness" factor and has increased with more advanced coding standards, as presented in Table-1. The Table-1 has the statistics of 5 minute sample trace of Star Wars-IV. If the burstiness factor is observed, the output of H.264/AVC encoder has the highest burstiness, when compared with that of H.264/SVC and MP4P2.

Multiplexing many such VBR sources greatly improves channel usage. SMG is a measure of channel usage when more than one video is multiplexed at a given link. Traffic smoothing is an inherent characteristic of multiplexing. However, better utilization of channel for a given number of multiplexed videos, is possible with an efficient multiplexing method employed in the multiplexer. Our candidates for experimentation are, High Quality, five minute, and frame- 
size traces of Star Wars-IV, encoded with H.264/SVC and H.264/AVC standards, with the coding pattern as G16B1.

Table-1: Burstiness statistics for the traces encoded with H.264 and MP4P2

\begin{tabular}{|l|l|l|l|l|l|l|}
\hline $\begin{array}{l}\text { Encoder } \\
\text { type }\end{array}$ & Min & Max & Mean & Variance & $\begin{array}{l}\text { Std. } \\
\text { Deviation }\end{array}$ & Burstiness \\
\hline H.264/SVC & 552 & $3.5269 \mathrm{E}+5$ & 64116.0 & $3.6132 \mathrm{E}+9$ & 60110.0 & 5.5 \\
\hline H.264/AVC & 184 & $3.2489 \mathrm{E}+5$ & 42813.0 & $2.1720 \mathrm{E}+9$ & 46605.0 & 7.5885 \\
\hline MP4P2 & 27760 & $4.4274 \mathrm{E}+5$ & 112040 & $1.7866 \mathrm{E}+9$ & 42268.0 & 3.9516 \\
\hline
\end{tabular}

\section{LITERATURE SURVEY}

Statistical Multiplexing of VBR video traffic has been extensively studied in the literature. The issues that were commonly addressed in the previous studies are modeling of VBR video multiplexer, estimation of average Channel utilization, estimation of average cell loss probability, performance analysis of statistical multiplexer using queuing models, statistical multiplexing gain, call admission control, effective bandwidth computation, modeling multiplexed traffic, throughput per source, call computation of representative bandwidth, etc.

Many of the previous studies [5, 11, 12], have used queuing processes and Markovian arrival processes, for modeling superposed variable bit rate video sources. The studies involved modeling the multiplexer and estimating the bandwidth requirements of the traffic based on the model. Multiplexer's performance was studied using a matrix analytic approach [8], with buffer occupancy and cell loss probabilities were estimated as a performance measure. Single server queuing models D-MMDP/D/1/k for modeling the ATM multiplexer was used in [10], and later bandwidth was estimated. Krunz et.al., [4,6] have assumed that cell per frame sequence of a video stream is a stationary stochastic process. The trace is arranged as a circular list and uses a random multiplexing technique. In another study, traffic envelope model was proposed using five parameters and proved that video sources can be statistically multiplexed with an effective bandwidth that is often less than the source peak rate. Poon et.al. [9] and Zhang et.al. [7] proposed a bandwidth estimation model to capture the characteristics of multiplexed highly correlated VBR video and used Chernoff boundary approximation for multiplexed traffic. According to Hiroshi et.al.,[13] burstiness of the multiplexed video sources depends on peak bit rate of individual sources and the time lags of the frame starting points between the video sources.

Recent studies involved estimation of Statistical Multiplexing Gain for homogeneous traffic sources. In [2], SMG is estimated as a Poisson distributed traffic stream. A recent study focused on multiplexing MPEG-4 FGS (Fine Granular Scalable) encoded traces and claims that the FGS traces are good candidates for multiplexing because of the two layer coding scheme (base and enhancement) involved. An FGS based frame-lag scheme was proposed in [3] which exploits intra and inter-layer correlations. Six basic multiplexing rules were proposed. Sarkar et.al.[1] used frame-aligned multiplexing method instead of random-multiplexing for better resource utilization, and then bandwidth requirements were estimated using multinomial model. A few studies were based on capping the VBR video, for consistent quality and better multiplexing gain [14]. By selectively choosing the target quality and the capping bit rate, more number of 
videos can be multiplexed on a given channel, and thus better multiplexing gain can be achieved.

Few studies [15] have experimented with combination of traffic grooming and multiplexing over two layer optical networks.

Most of the studies have focused on modeling the statistical multiplexer with a buffer using Markovian, queuing models and then used the models for bandwidth estimation. It is evident that the cost and the complexity of implementation have restricted their usage to theoretical studies. Moreover, the models were based on a particular encoding standard and thus have not served the purpose of multiplexing in general. Added to that, not many of the studies have used the recent standards like H.264/SVC, H.264/AVC for their experimentation.

We can observe that, the techniques were basically mathematical models that were used to estimate various parameters and efficiency of statistical multiplexers, with bandwidth estimation carried out using the underlying model.

\section{EXISTING METHODOLOGIES}

In this section a few of the practical methods were presented.

\subsection{Frame-lag based multiplexing}

Assume that there are $\mathrm{k}$ videos, say $\mathrm{V}_{0}, \mathrm{~V}_{1}, \mathrm{~V}_{2}, \ldots \ldots \ldots, \mathrm{V}_{\mathrm{k}-1}$ in the multiplexed system. The start of a video $\mathrm{V}_{\mathrm{m}}$ where $\mathrm{m} \in\{0, \ldots ., \mathrm{k}-1\}$, is delayed by ' $\mathrm{m}$ ' frames.

This technique is quite simple and does not need any complex analysis for understanding. This involves less implementation costs. However the collision of I, P, and B frames is inevitable when the cycle repeats.

\subsection{Random multiplexing}

Here a random number is initially generated for each of the videos in the multiplexed system, to determine the starting point for each video, and then incremented by one frame going in cyclic order to complete. Even using a seed, the randomness involved might lead to less smoothed traffic.

\subsection{Frame-aligned multiplexing}

This technique was introduced to see that the number collisions of each of the I, P, or B frame types are reduced to the minimum.

It is a variation of random multiplexing, where the starting random positions are incremented by fewest possible frames such that $\mathrm{i}^{\text {th }}$ video starts at the $\mathrm{i}^{\text {th }}$ frame type in the underlying GOP sequence.

This technique seems to be a better technique as the number of I or P or B frames collisions are reduced to the minimum. Each video starts at a random position. Increment for each video varies based on the starting frame type, thus involving huge implementation costs.

\subsection{Traffic-Shifting method (for self-similar sources)}

This method presented in [16], is exclusively for self similar traffic with Hurst parameter greater than 0.5. As per this method, some aggregate of the incoming traffic sources is to be computed first. Then a rate control $R_{c}$ for the traffic is assumed. If the aggregation is greater than $R_{c}$ then shift the member by one or delay its transmission. Hurst parameter was persistent in the multiplexed traffic. 


\subsection{Shuffle method (for self-similar sources)}

According to this method presented in [16], traffic is divided into blocks of some time range and then shuffled. But the structure of time series inside a block remains unaltered. Hurst parameter and ACF were addressed as indicative of reduced burstiness.

\section{THE NEW METHOD (ERA)}

In this section we present a methodology by name "ERA multiplexing". This method uses a spiral-linear indexing procedure that is not only simple to implement and also avoids collisions of same frame types.

Sampling each video in the multiplexed system starts at an initial random position which is chosen such that the it lies in the proximity of the median of the number of frames, and then is incremented and decremented alternatively on either side till the spiral is complete, and if any of the frames are on excess on either side of the initial random position, they are either incremented by one or decremented by one, depending on the side of excess frames. This process is repeated for each individual video in the multiplexed system. Finally frame-lag scheme is applied on the resultant jumbled videos.

Formally, let ' $x$ ' be the random position chosen in the proximity of the median of a given video trace. Also, let $l=x-1$; and $\mathrm{r}=|\mathrm{v}|-x$, where $|\mathrm{v}|$ is the length of the video trace.

Now the frames are selected in the following sequence of positions

$x, x+1, x-1, \ldots, x+\mathrm{i}, x-\mathrm{I}, \ldots, x-\mathrm{l}, x+\mathrm{l}+1, x+\lfloor+2, \ldots, x+\mathrm{r}$, for $\mathrm{l}<\mathrm{r}$ and

$x, x+1, x-1, \ldots, x+\mathrm{i}, x-\mathrm{i}, \ldots, x+\mathrm{r}, x$-r, $x$-r-1, $x$-r- $2, \ldots, 1$, for $\mathrm{r}<\mathrm{l}$

In eqn., (1) $x, \ldots, x-\lfloor$ is spiral and $x+\downarrow+1 \ldots, x+\mathrm{r}$ is a linear strand. Similarly in eqn.,(2) $x, \ldots, x-\mathrm{r}$ is spiral and $x-\mathrm{r}-1, \ldots, 1$ is a linear strand and for $l=\mathrm{r}$, we get a spiral without a linear strand.

\section{Algorithm 1: (ERA)}

//input:

$\mathrm{g} \leftarrow$ range;

$x \leftarrow$ Random

(median-g/2, median+g/2)

//output: multiplexed file

Begin:

$$
\begin{aligned}
& \text { print }(x) ; \\
& \mathrm{l} \leftarrow x-1 ; \\
& \mathrm{r} \leftarrow \mathrm{v} \mid-x ; \\
& \mathrm{i} \leftarrow 1 ; \\
& \text { if } \quad \mathrm{L}<\mathrm{r} \text { then } \\
& \text { while }((x-\mathrm{i}) \geq 1) \text { do } \\
& \quad \text { print }(x+\mathrm{i}) ; \\
& \quad \text { print }(x-\mathrm{i}) ; \\
& \mathrm{i} \leftarrow \mathrm{i}+1 ; \\
& \text { endwhile } \\
& \text { while }((x+\mathrm{i}) \leq \mid \mathrm{vl}) \text { do } \\
& \text { print }(x+\mathrm{i}) ; \\
& \mathrm{i} \leftarrow \mathrm{i}+1 ;
\end{aligned}
$$




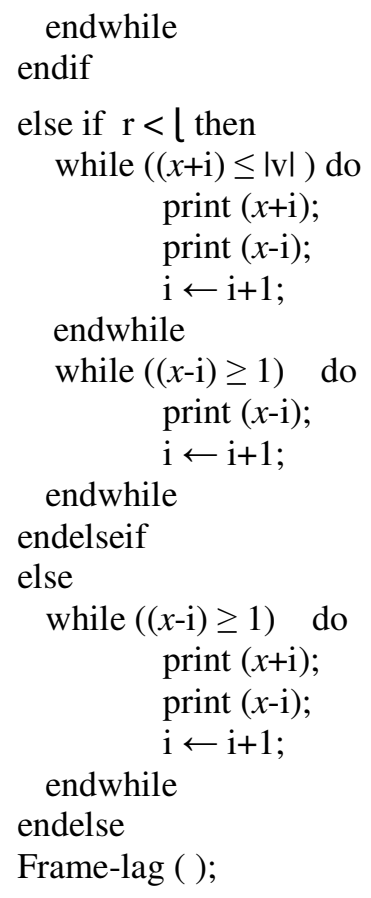

End:

We have successfully implemented this technique and compared the smoothness and statistical multiplexing gain for the resultant traffic with that of frame-aligned multiplexing technique, and frame-lag technique. This technique is well behaved, efficient and faster and thus can be used for general multiplexing.

\section{EXPERIMENTAL SETUP}

We assume that our multiplexer is devoid of any buffer. We have used 5 minute high quality, single layer, CIF traces of Star Wars-IV, encoded with H.264/SVC and H.264/AVC at a frame rate of 30 frames per second, on the pattern G16B1. The traces were obtained from [17]. So there are 9000 frames in a 5 minute trace. The statistics of the trace encoded with H.264/SVC and H.264/AVC are given in Table-1. Frame size data is converted to cell data, and the number of cells per frame, is taken for experimentation. The cell-size histograms are shown in below in figures 1, 2, and 3 for H.264/SVC, H.264/AVC, and MP4P2 respectively.

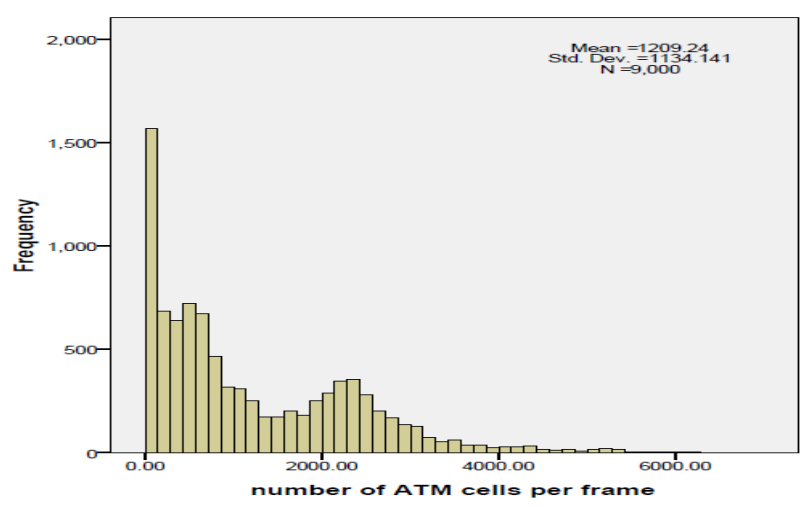

Figure 1. Cell-size histogram (H.264/SVC) 


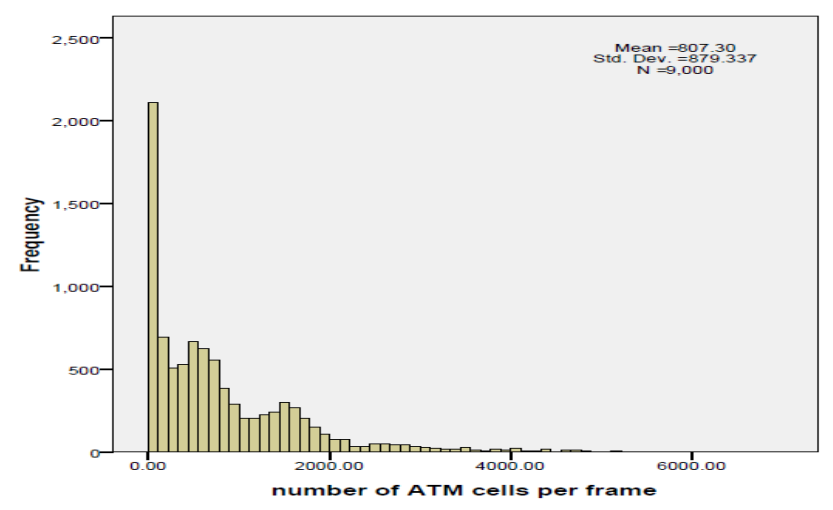

Figure 2. Cell-size histogram (H.264/AVC)

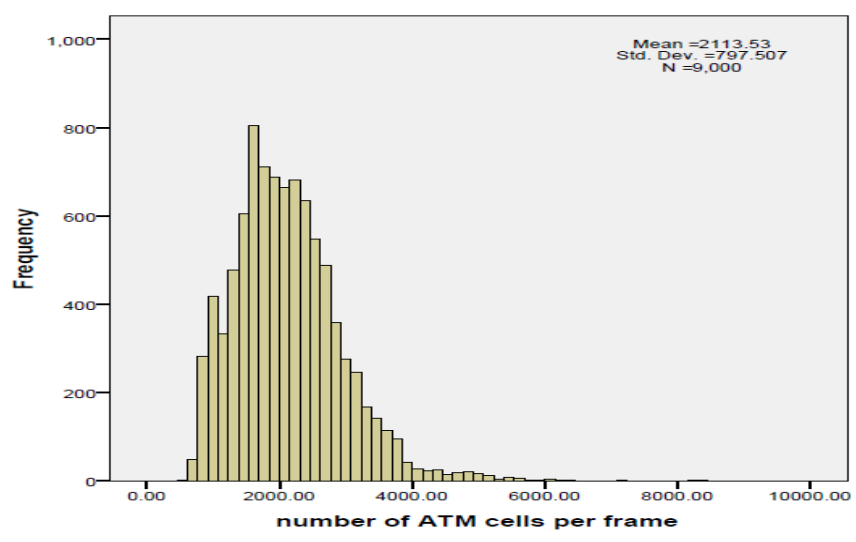

Figure 3. Cell-size histogram (MP4P2)

\section{RESULTS AND ANALYSIS}

We have compared our results with Frame-aligned multiplexing and Frame-lag multiplexing techniques. We have implemented homogeneous multiplexing [1], where the bandwidth requirements of the videos in the multiplexing system, are the same. Multiplexing is applied on the same copy of Star-Wars IV. Also heterogeneous multiplexing, where the bandwidth requirements of the videos in the multiplexing system, are not the same. We found that the multiplexed output of our technique is much smoother than that of the other techniques.

The figures 4 and 5 are burstiness and SMG against number of multiplexed videos for H.264/SVC traces. The graphs indicate that our technique is superior to other techniques in terms of traffic smoothing as evident from reduced burstiness, and thereby better multiplexing gain. SMG is estimated using the ratio of sum of peak input rate to that of peak output rate. The results indicate that statistical multiplexing gain is considerably higher for our technique when compared with that of others. Figures 6 and 7 are burstiness and SMG curves for H.264/AVC traces. 


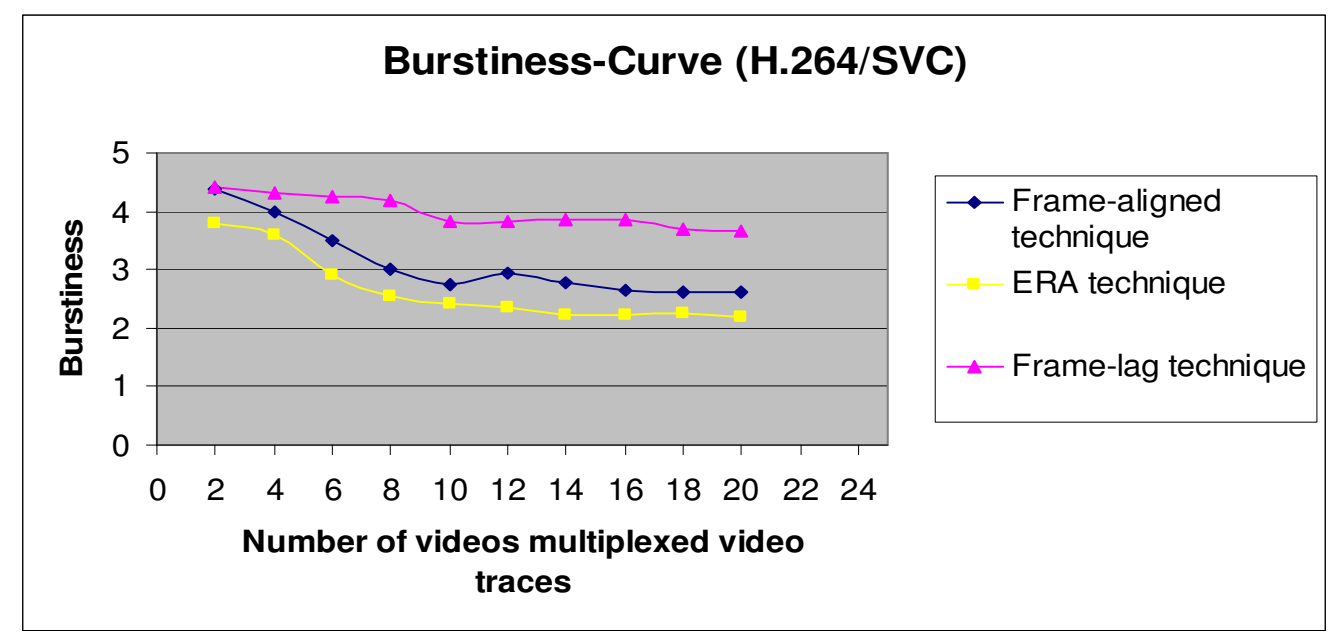

Figure 4. Number of multiplexed traces Vs. Burstiness (H.264/SVC)

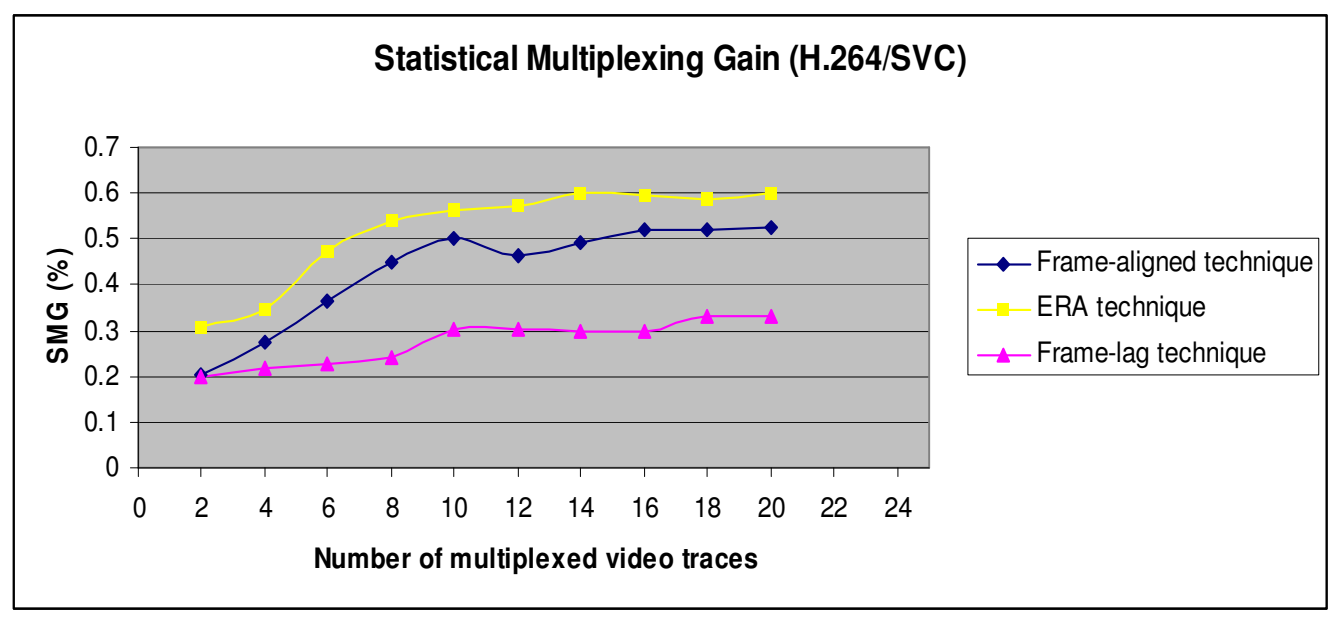

Figure 5. Number of multiplexed traces Vs. Statistical Multiplexing Gain (H.264/SVC)

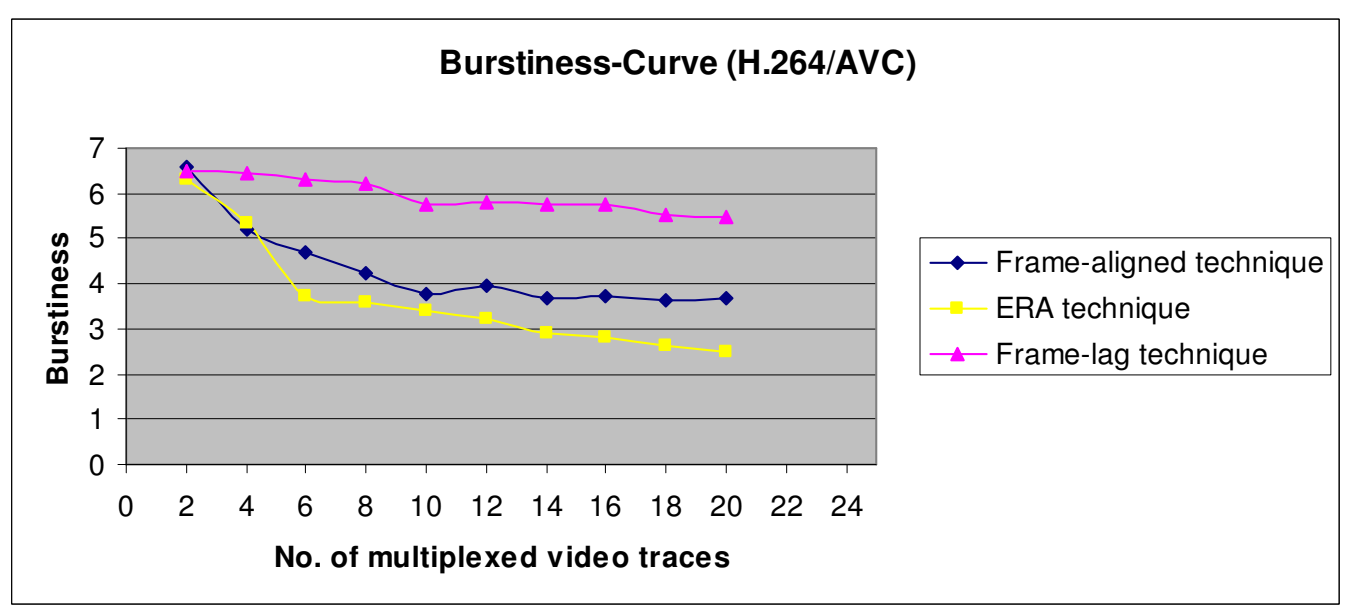


Figure 6. Number of multiplexed traces Vs. Burstiness (H.264/AVC)

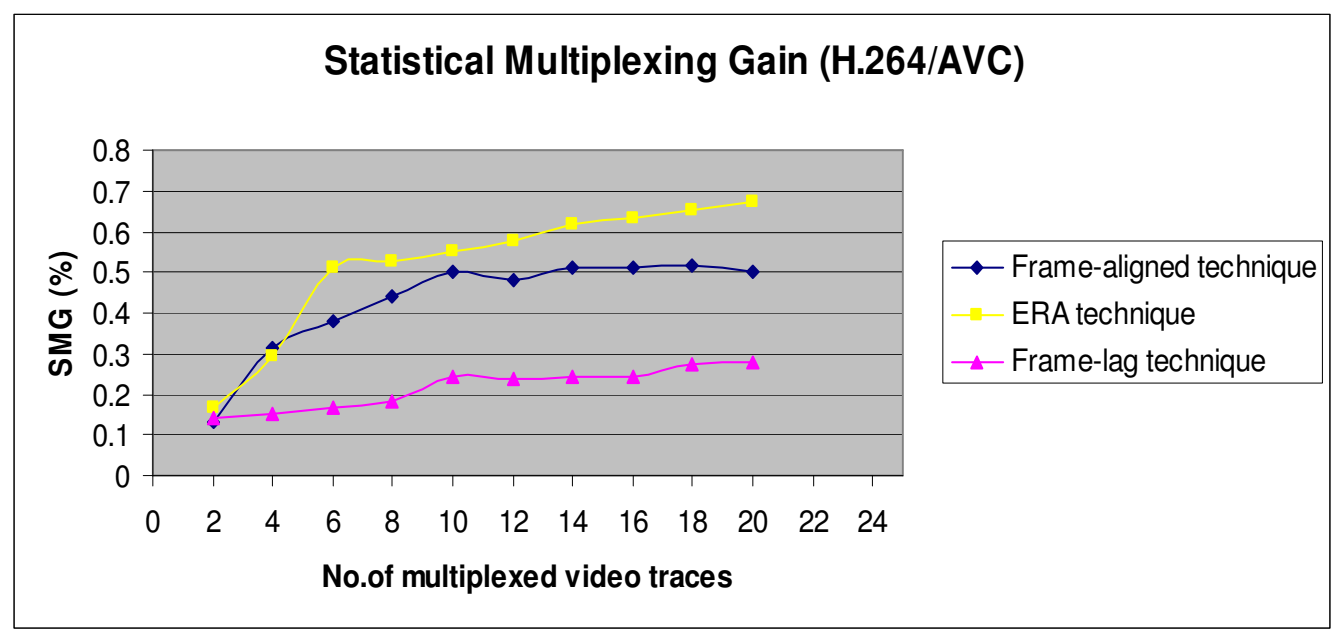

Figure 7. Number of multiplexed traces Vs. Statistical Multiplexing Gain (H.264/AVC)

Table-2: Some cases and statistics of Heterogeneous traffic

\begin{tabular}{|c|c|c|c|c|c|}
\hline \multirow[t]{2}{*}{ Case } & \multirow[t]{2}{*}{ Heterogeneous Mix } & \multicolumn{4}{|c|}{ Burstiness } \\
\hline & & Original & $\begin{array}{l}\text { Frame- } \\
\text { aligned }\end{array}$ & $\begin{array}{l}\text { Frame- } \\
\text { lag }\end{array}$ & ERA \\
\hline (I) & $\begin{array}{l}\mathrm{HQ} \quad(2) \quad-\mathrm{QP} \quad(10)+\mathrm{MQ} \\
\mathrm{QP}(28)+\mathrm{LQ}(2)-\mathrm{QP}(48)\end{array}$ & 6.4698 & 4.1953 & 4.1032 & 3.8287 \\
\hline (II) & $\begin{array}{l}\text { HQ-H.264/SVC(2)+HQ-H.264/AVC } \\
\text { (2)+HQ MP4P2-HQ(2) }\end{array}$ & 5.1163 & 2.3214 & 2.4292 & 2.6697 \\
\hline (III) & $\begin{array}{l}\text { HQ-H.264/SVC(3)+HQ-H.264/AVC } \\
\text { (3) }\end{array}$ & 6.3367 & 3.3279 & 3.3747 & 2.5865 \\
\hline
\end{tabular}

We have conducted experiments using traces of different qualities. We have taken two each of low quality, medium quality and high quality traces of Star Wars-IV.

Table-2 indicates that even when there is a heterogeneous mix of videos, ERA technique gave a better result, in terms of comparatively lower burstiness, as is evident from results of the first case, where a total of 6 traces were multiplexed, with two each from three different qualities (High, medium, and low) of Star-Wars IV, 5 minute traces [17]. The Quantization parameter is given in as QP with the value in brackets as shown in Table-2. But the results of the second case indicate a comparatively meager performance by our method, as is evident from the burstiness values. It is more obvious that, when a better encoding method line H.264 is available, one would not prefer to stick to the old encoding methods like MPEG-4, and hence we would like to justify that, the second case becomes a rare occurrence. The third case again shows a better result with our method.

The burstiness of the input traces in the multiplexed system is computed as the ratio of the sum of the peaks to the sum of the means of the traces. 
Figures 8 and 9 present the correlation structure in the multiplexed trace file when same copies of 12, H.264/SVC encoded, and 12, H.264/AVC Start Wars-IV trace files, respectively, are multiplexed. Our method shows a persistent correlation structure. But for multiplexed trace files the correlation structure is less significant.

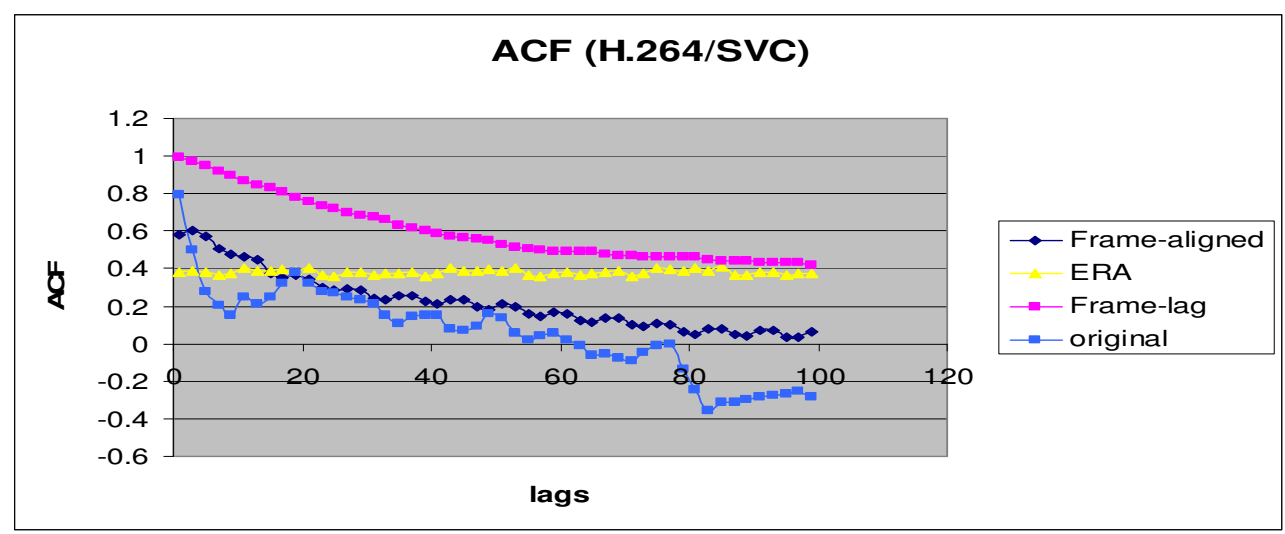

Figure 8. Auto-Correlation function (H.264/SVC)

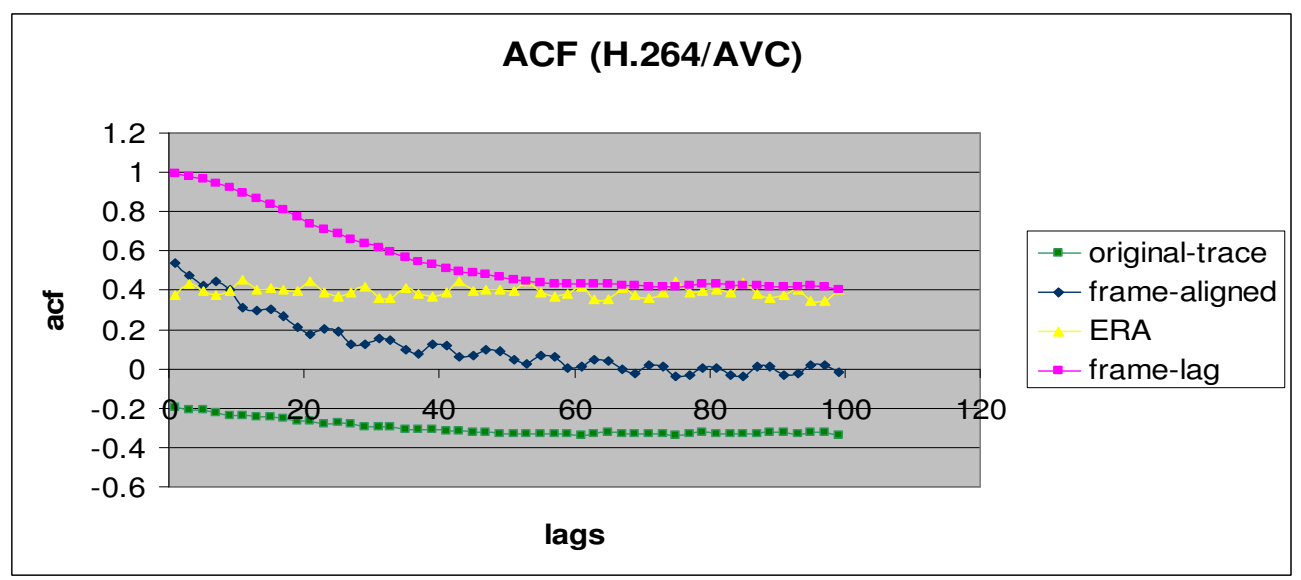

Figure 9. Auto-Correlation function (H.264/AVC)

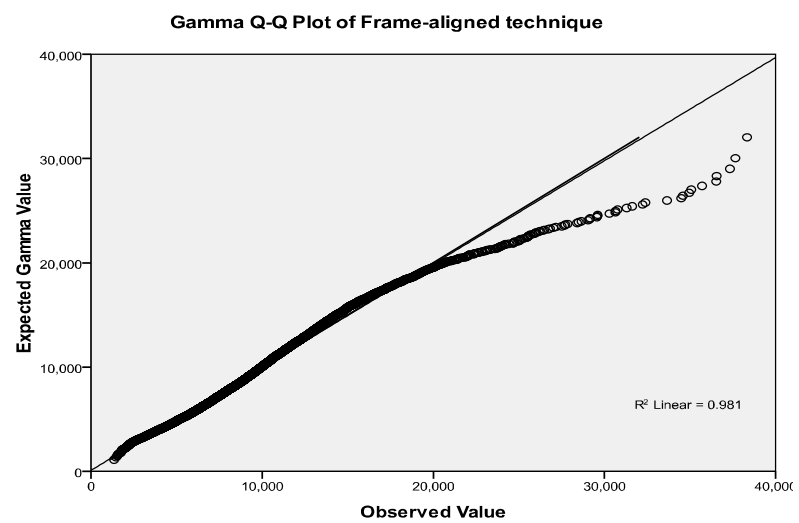

Figure 10. Quantile-Quantile plot of Gamma model vs. observed values for Frame-aligned technique 


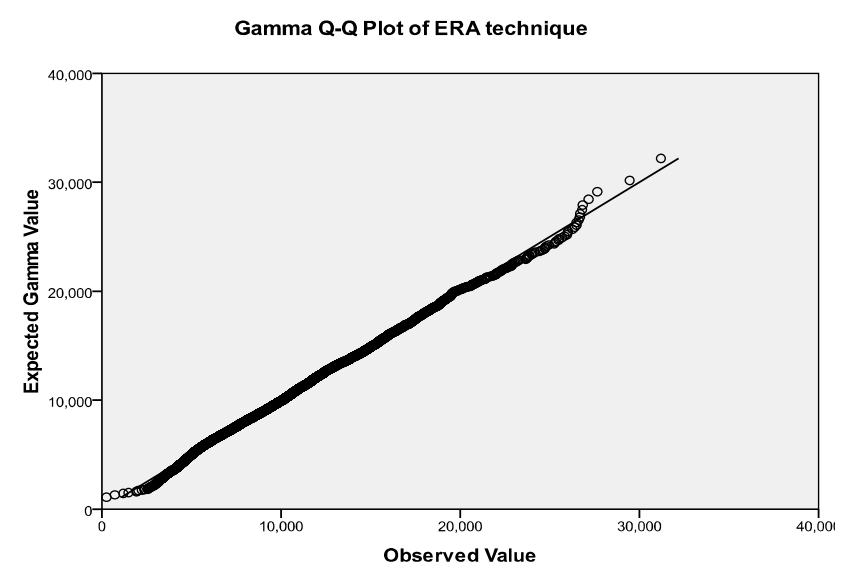

Figure 11. Quantile-Quantile plot of Gamma model vs. observed values for ERA technique

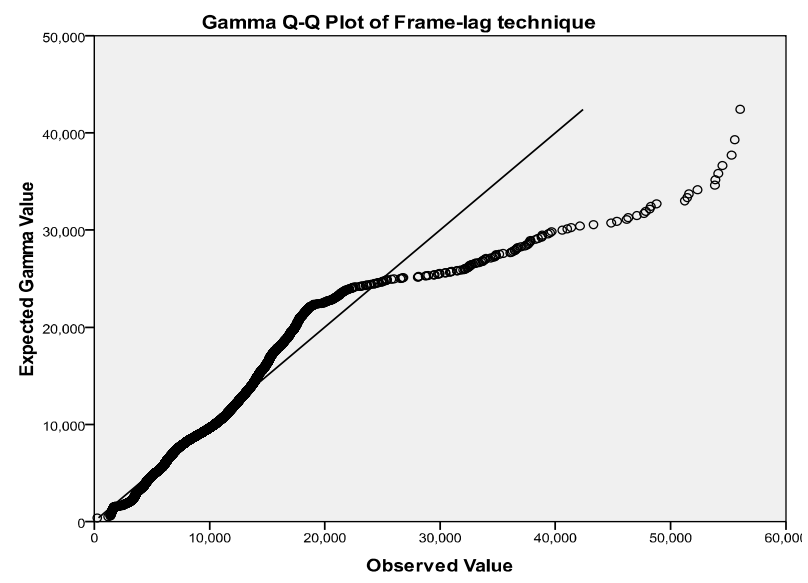

Figure 12. Quantile-Quantile plot of Gamma model vs. observed values for Frame-lag technique

Figures 10, 11 and 12 throw insight into another special characteristic of our technique. It is a challenging task to model the multiplexed output, as the output trace has no consistent behavior and moreover, based on the multiplexing technique the characteristics of the output change dramatically. It was observed that the multiplexed output could be modeled using the distributions like, Gamma, Lognormal and pearson-5 in most of the cases [1]. We have presented here a case, when 12 multiplexed traces (homogeneous) were used, where we have used Gamma distribution to compare the Quantile-Quantile plots of the output aggregate traces of H.264/AVC, High-Quality using the three techniques.

\section{CONCLUSIONS}

We have conducted experiments on high quality traces of Star Wars-IV encoded with H.264/SVC, H.264/AVC and occasionally MP4P2 and our results show that burstiness of the multiplexed trace is comparably lesser with our method, resulting in a much smoother traffic. Our method also assumes that the multiplexer is devoid of buffer. Bandwidth allocation is much easier and Statistical multiplexing gain is better. This method is easy to implement and the 
complexity is $\mathrm{O}(n * k)$, where $n$ is the maximum number of frames in a video and $k$ the number of videos.

We have to conduct experiments further with traces of different activity rates. The experiments are to be conducted on traces of longer duration. Bandwidth estimation should be carried out.

\section{ACKNOWLEDGEMENTS}

The authors would like to thank all those who have extended their support for successful completion of this work.

\section{REFERENCES}

[1] D. Sarkar, U. K. Sarkar, and W. Zhou, (2007) "Bandwidth estimation for multiplexed videos using multinomial model," Computer Communications, vol. 30, no. 2, pp. 269-279.

[2] Khalaf, G.A.F.M., El-Yamany, S.S.K. (2001), "Statistical multiplexing gain: direct estimation and itsapplication to admission control in ATM networks", Proceedings of the Eighteenth National Radio Science Conference, Volume 2, Issue 1, Page(s):483 - 496.

[3] Xiaokang Yang, Nam Ling, (2006) "Statistical Multiplexing based on MPEG-4 Fine Granularity Scalability Coding", Journal of VLSI Signal Processing Systems, Volume 42, Issue 1, Pages: $69-77$.

[4] M. Krunz and S.K. Tripathi, (1997) "Impact of Video Scheduling on Bandwidth Allocation for Multiplexed MPEG Streams," Multimedia Systems, vol. 5, pp. 347-357, ACM/Springer.

[5] Ohta, C. Shinagawa, K. Onozato, Y., (1996)" Cell Loss Properties for Multiplexing of MPEG Video Sources Considering Picture Coding Types in ATM Networks", IEEE International Conference on Converging Technologies for Tomorrow's Applications", Volume: 3, page(s) 1396-1400.

[6] M. Krunz, R. Sass, and H. Hughes, (1995) "Statistical Characteristics and Multiplexing of MPEG Streams,” Proc. IEEE INFOCOM., pp. 455-462.

[7] Zhi-Li Zhang; Kurose, J.; Salehi, J.D.; Towsley, D.,(1997)” Smoothing, statistical multiplexing, and call admission control forstored video", IEEE Journal on Selected Areas in Communications, Volume 15, Issue 6, Page(s): 1148 - 1166.

[8] Blondia, C.; Casals, O.,(1992)" Performance analysis of statistical multiplexing of VBR sources", Proceedings of the eleventh annual joint conference of the IEEE computer and communications societies, vol.2, Pages: $828-838$.

[9] W. C. Poon and K. T. Lo,(1999)" The study on statistical multiplexing of homogeneous VBRMPEG video streams", Computer Communications 22 (15-16): 1457-1467.

[10] Ni, J.; Yang, T.; Tsang, D.H.K.,(1996) “ Source modelling, queueing analysis, and bandwidth allocation forVBR MPEG-2 video traffic in ATM networks", IEE Proceedings in Communications, Volume 143, Issue 4, Page(s): 197 - 205.

[11] G. Ramamurthy B. Sengupta,(1992)"Modeling and analysis of a variable bit rate video multiplexer", Proceedings of the eleventh annual joint conference of the IEEE computer and communications societies, Vol.2.

[12] Maglaris, B. Anastassiou, D. Sen, P. Karlsson, G. Robbins, J.D., (1998) "Performance models of statistical multiplexing in packet videocommunications", IEEE Transactions on Communications, Volume: 36, Issue: 7, page(s): 834-844.

[13] Hiroshi Saito, Mashatoshi Kawarazaki, Hiroshi Yamada, (1990)" An analysis of statistical multiplexing in an ATM transport network", Proceedings of IEEE International Conference on Communications, Vol.2, page(s): 478-482.

[14] Z. Avramova1, D. De, Vleeschauwer1,2, S. Wittevrongel1, H. Bruneel1, (2005)"Multiplexing Gain of Capped VBR Video", Workshop on QoS and Traffic Control”. 
[15] Kern, A. Somogyi, G. Cinkler, T.,(2006) "On the Gain of Statistical Multiplexing over Traffic Grooming”, International Conference on Transparent Optical Networks, Volume: 3, pages: 112115 .

[16] Linawati Sastra, N.P., (2008) "Statistical multiplexing strategies for self-similar traffic", International Conference on Wireless and Optical Communications Networks, page(s): 1-5.

\section{Authors}

K. S. Easwarakumar is Professor and Head at the Department of Computer

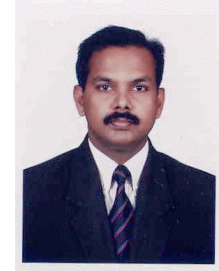
Science and Engineering at Anna University, Chennai, India. He received his M.Tech in Computer and Information Sciences from Cochin University of Science and Technology, Cochin and Ph.D in Computer Science and Engineering from Indian Institute of Technology, Madras. His research interests include parallel and distributed computing, Data Structures and Algorithms, Graph Algorithms, Parallel Algorithms, Computational Geometry, Theoretical Computer Science and Molecular computing.

T.Raghuveera is Senior Lecturer, pursuing his

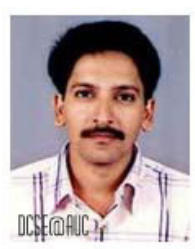
doctoral degree at the Department of Computer Science and Engineering at Anna University, Chennai, India. He received his Masters degree in Multimedia development from Jadavpur University, Calcutta. His research interests include, VBR video traffic modelling, multiplexing, network resource management, traffic prediction etc., 